Artículo

\title{
Impacto del cambio climático en la distribución potencial de Tithonia diversifolia (Hemsl.) A. Gray en México
}

Noé Durán Puga ${ }^{\text {a }}$

José Lenin Loya Olguín b

José Ariel Ruiz Corral ${ }^{a^{*}}$

Diego Raymundo González Eguiarte ${ }^{a}$

Juan Diego García Paredes b

Sergio Martínez González b

Marcos Rafael Crespo González ${ }^{\text {a }}$

${ }^{a}$ Universidad de Guadalajara. Centro Universitario de Ciencias Biológicas y Agropecuarias. Camino Ramón Padilla Sánchez No. 2100 Nextipac, 44600, Zapopan, Jalisco, México.

${ }^{\mathrm{b}}$ Universidad Autónoma de Nayarit. Unidad Académica de Agricultura. Carretera TepicCompostela, km 9. Xalisco, Nayarit, México.

*Autor de correspondencia: ariel.ruiz@academicos.udg.mx

\section{Resumen:}

El objetivo fue estimar el posible impacto del cambio climático en la distribución potencial de $T$. diversifolia en México. Nichos de distribución fueron modelados con MaxEnt para la climatología de referencia 1951-2000, y dos escenarios de cambio climático 2041-2060 y 2061-2080, considerando 20 variables bioclimáticas y dos topográficas. Para las climatologías futuras se consideró el modelo de circulación global (MCG) HadGEM2-ES, con dos vías representativas de concentración de gases efecto invernadero (rcp4.5 y rcp8.5). Esta información se obtuvo de la página web Global Climate Data de WorldClim y se trabajó con el sistema Idrisi Selva como imágenes raster con una resolución de 2.5 min de arco. Las 
variables que más contribuyeron a explicar la distribución geográfica de $T$. diversifolia fueron la precipitación acumulada mayo-octubre (pa5-10) y la temperatura máxima media del mes más cálido (txmc). El umbral del percentil 10th de la presencia logística predice una superficie (para la climatología de referencia 1951-2000), que representa el $30.71 \%$ de la extensión de México. La modelación de nichos en los diversos escenarios climáticos registra zonas de expansión, así como zonas de retracción para la distribución potencial de la especie, pero después de hacer un balance, se obtiene que las áreas potenciales para T. diversifolia se incrementarán ligeramente con respecto a la climatología de referencia 1950-2000, con valores estimados de $31.62 \%, 31.83 \%, 32.45 \%$ y $32.45 \%$ del territorio mexicano en los escenarios 2041-2060 rcp8.5, 2041-2060 rcp4.5, 2061-2080 y 2061-2080 rcp4.5 y rcp8.5, respectivamente. Se concluye que el cambio climático traería más beneficios que restricciones para la dispersión de T. diversifolia.

Palabras clave: Cambio climático, Tithonia diversifolia, Descriptores ecológicos, Nichos de distribución.

Recibido: 20/11/2017

Aceptado: 04/07/2018

\section{Introducción}

La producción de alimentos se está convirtiendo en un verdadero reto en un clima que está cambiando. Bajo este contexto, la diversificación de la alimentación animal es un aspecto clave para adaptarse mejor al cambio climático. Este es el motivo para evaluar el impacto del cambio climático sobre la presencia y distribución potencial de las especies forrajeras. Este es el caso específico de $T$. diversifolia, nativa y con distribución actual en las tierras bajas del sudeste de México, Centro y Sudamérica, especie que se considera un importante recurso genético y una planta excepcional como alternativa en la alimentación animal ${ }^{(1,2)}$. El riesgo futuro para muchas especies de plantas debido al cambio climático no ha sido bien establecido aún.

La temperatura global ha aumentado $0.85{ }^{\circ} \mathrm{C}$ en el periodo de 1901 al $2011^{(3)}$. Se ha establecido sobre la base de los modelos de predicción del clima, que la presión de saturación del vapor ha sido muy sensible a los cambios de temperatura, por lo que se espera que se manifiesten futuras rupturas en el ciclo global del agua ${ }^{(4)}$. Por otro lado, se ha mencionado que la disponibilidad de agua presentará una marcada reducción anual en el sureste de Estados Unidos de Norteamérica, el Caribe y diversas partes de México ${ }^{(5)}$. Además, la agricultura se expande e intensifica principalmente en los trópicos ${ }^{(6)}$; se estima que alrededor 
del $80 \%$ de la nueva tierra de cultivo será a base de sustitución de bosques ${ }^{(7)}$. Por lo tanto, al retrasar o retener la expansión de la agricultura en los trópicos se reducirán las emisiones de carbono y la pérdida de la biodiversidad ${ }^{(8)}$.

Con el cambio climático, la diversidad de climas puede ser modificada, así como la composición de los ecosistemas ${ }^{(9)}$, que incluirían alteraciones en la distribución, fenología y un aumento de riesgo de especies en peligro de extinción ${ }^{(10)}$. Se considera que varios componentes del cambio climático afectarán a todos los niveles de la biodiversidad; es decir, desde los organismos y poblaciones hasta las áreas bióticas ${ }^{(11)}$. En los niveles básicos, el cambio climático es capaz de disminuir la diversidad genética en las poblaciones debido a una selección direccional y una migración dinámica, que podría interferir en la capacidad de resiliencia y la funcionalidad de los ecosistemas ${ }^{(12)}$; por lo tanto, podría causar la modificación de la red de interacciones a nivel de la comunidad ${ }^{(13)}$. Además, un efecto del cambio climático podría inducir la invasión de especies potencialmente peligrosas para un ecosistema $^{(14)}$; afectando la fisiología, fenología y el comportamiento de especies ${ }^{(15)}$.

De acuerdo con lo anterior, México está interesado en la construcción de un inventario en lo que respecta a los efectos previstos del cambio climático en las especies silvestres deseables. Debido a eso, el objetivo fue estimar el impacto del cambio climático en la distribución potencial de $T$. diversifolia.

De acuerdo con diferentes autores los usos reportados de Tithonia incluyen producción de forrajes, control de erosión del suelo, como material de construcción y un refugio para las aves $^{(16)}$. Como contrapartida $T$. diversifolia es considerada como una hierba alelopática con aleloquímicos solubles en agua en partes de la planta y con tal potencia fitotóxica, que podría suprimir el crecimiento y la acumulación de nutrientes en cultivos asociados ${ }^{(17)}$.

\section{Material y métodos}

\section{Base de datos}

Se consideraron los datos de 52 sitios de población de T. diversifolia, con distribución actual en las tierras bajas del sudeste de México ${ }^{(1,2)}$. Las bases de datos se obtuvieron a partir de dos fuentes: Inventario Nacional Forestal y de Suelos (INFyS) de la Comisión Nacional Forestal de México (CONAFOR) y del sitio http://www.tropicos.org/.

\section{Áreas potenciales de distribución}

En este estudio se utilizó el modelo de MaxEnt (máxima entropía) ${ }^{(18)}$ para modelar el nicho ecológico y predecir la distribución geográfica más probable de T. diversifolia. MaxEnt ha 
sido ampliamente utilizado para estimar distribuciones potenciales de plantas y animales con alta precisión; algunos grupos terrestres son excelentes ejemplos ${ }^{(19)}$.

En el modelo MaxEnt la distribución de una especie se representa mediante una función de probabilidad $\mathrm{P}$ sobre un conjunto $\mathrm{X}$ de sitios en el área de estudio. Se construye un modelo de $\mathrm{P}$ mediante un conjunto de restricciones derivado de datos empíricos de presencia de la especie $^{(20)}$. Las restricciones se expresan como funciones simples de variables ambientales conocidas. El algoritmo de MaxEnt obliga a que la media de cada función de cada variable se aproxime a la media real en las áreas de presencia de la especie. De todas las posibles combinaciones de funciones, se selecciona la que logra minimizar la función de entropía; la cual es medida con el índice de Shannon. La expresión general de la función de probabilidad para $i$ variables ambientales es ${ }^{(21)}$ :

$$
P(x)=e^{\lambda \cdot f(x)} / Z \lambda
$$

Donde:

$\mathbf{P}(\mathbf{x})=$ función de probabilidad;

$\lambda=$ vector de coeficientes de ponderación;

$\boldsymbol{f}=$ vector correspondiente de funciones de variables ambientales;

$\mathbf{Z}=$ constante de normalización para asegurar que $\mathrm{P}(\mathrm{x})=1$.

Los valores $\mathrm{P}(\mathrm{x})$ así obtenidos, representan valores de idoneidad relativa para la presencia de la especie, constituyendo así la base de un modelo de distribución potencial. Un alto valor de la función de distribución en cada píxel indica que tiene condiciones favorables para la especie $^{(22)}$.

En este estudio con el uso de capas de parámetros ambientales y los datos de presencia de especies, se generó un modelo. El $75 \%$ de los registros de presencia se utilizaron como puntos de entrenamiento y $25 \%$ como puntos de validación, además se utilizó el índice AUC (área bajo la curva), para evaluar el modelo estadístico, ya que este índice es de los más utilizados para medir la calidad de los modelos ${ }^{(20)}$. Los ajustes que se pueden determinar en el modelo de MaxEnt, afectan su precisión, ya que el modelo puede trabajar con variables ambientales simples o complejas.

\section{Bases de datos y parámetros ambientales}

Los datos mensuales, estacionales y anuales de precipitación, temperatura máxima, temperatura mínima, temperatura media y oscilación térmica de los períodos 1950-2000 (climatología de referencia) y 2041-2060 y 2061-2080 (climatología futura), se utilizaron para una caracterización ambiental de los sitios de presencia de las especies. Estos datos 
climáticos se obtuvieron del portal Global Climate Data de WorldClim y las imágenes se trabajaron en formato ascii y raster, con una resolución de $2.5 \mathrm{~min}$ de arco. Para los períodos 2041-2060 y 2061-2080, el MCG HadGEM2-ES fue considerado con dos vías de concentración representativas de gases de efecto invernadero: Rcp 4.5 y Rcp 8.5. Este modelo ha sido utilizado en los nuevos escenarios de cambio climático para México; estudio presentado en el quinto informe de evaluación sobre el cambio climático IPCC (Grupo Intergubernamental de Expertos sobre el Cambio Climático) ${ }^{(23)}$. Otras variables que se incluyeron en la modelación de la distribución de T. diversifolia, fueron la altitud y la pendiente del suelo, imágenes que se obtuvieron del Sistema Nacional de Información Ambiental (SIAN) del Instituto Nacional de Investigaciones Forestales Agrícolas y Pecuarias $^{(24)}$. La lista de variables utilizadas para el modelo de nicho espacial fueron: pendiente del suelo $(\%)$, altitud $(\mathrm{m})$, temperatura máxima media anual (Txan), temperatura máxima media de mayo a octubre (tx5-10), temperatura máxima media noviembre-abril (tx11-4), temperatura máxima del mes más cálido (txmc), temperatura media anual (tman), temperatura media de mayo a octubre (tm5-10), temperatura media noviembre-abril (tm114), temperatura mínima del mes más frío (timf), temperatura media del mes más frío (tmmf), temperatura mínima promedio anual (tian), temperatura media mínima de mayo a octubre (ti5-10), temperatura media mínima noviembre-abril (ti11-4), precipitación acumulada anual (pa), precipitación acumulada mayo a octubre (pa5-10) precipitación acumulada noviembreabril (pa11-4), precipitación acumulada del mes más seco (pms), precipitación del mes más húmedo (pmh), oscilación térmica media anual (otan), oscilación térmica de mayo a octubre (ot5-10) y oscilación térmica noviembre a abril (ot11-4).

\section{Áreas con probabilidad de aptitud ambiental}

El modelo obtenido de predicción MaxEnt para la distribución potencial de T. diversifolia fue examinado con el sistema de Idrisi Selva ${ }^{(25)}$, y se hizo un mapa con los valores del umbral de los píxeles correspondientes al percentil $10^{(26)}$. Para el cálculo de la superficie potencial de distribución de la especie, las áreas ocupadas por las masas de agua y los centros urbanos no fueron consideradas. Estas capas temáticas se obtuvieron mediante la manipulación del uso del suelo y la capa de vegetación ${ }^{(27)}$.

\section{Descriptores ecológicos}

Los descriptores ecológicos para $T$. diversifolia se determinaron sobre la base de los intervalos ambientales derivados de la caracterización de los parámetros de sitios de presencia de la especie. Esto se hizo utilizando el sistema IDRISI y con las imágenes raster de cada variable ambiental y las coordenadas geográficas de cada sitio de presencia. 


\section{Resultados y discusión}

En el modelo del nicho de distribución potencial de T. diversifolia, la curva operacional mostró resultados satisfactorios (Figura 1). Al considerar los datos de entrenamiento (75\%), el área bajo la curva (AUC) de T. diversifolia fue de 0.979; mientras que cuando se utilizan los datos de prueba (25\%), la validación del modelo reportó una AUC de 0.966, lo que indica que la capacidad del modelo para representar la presencia de especies fue satisfactoria ${ }^{(28)}$.

Figura 1. Curva operacional para T. diversifolia

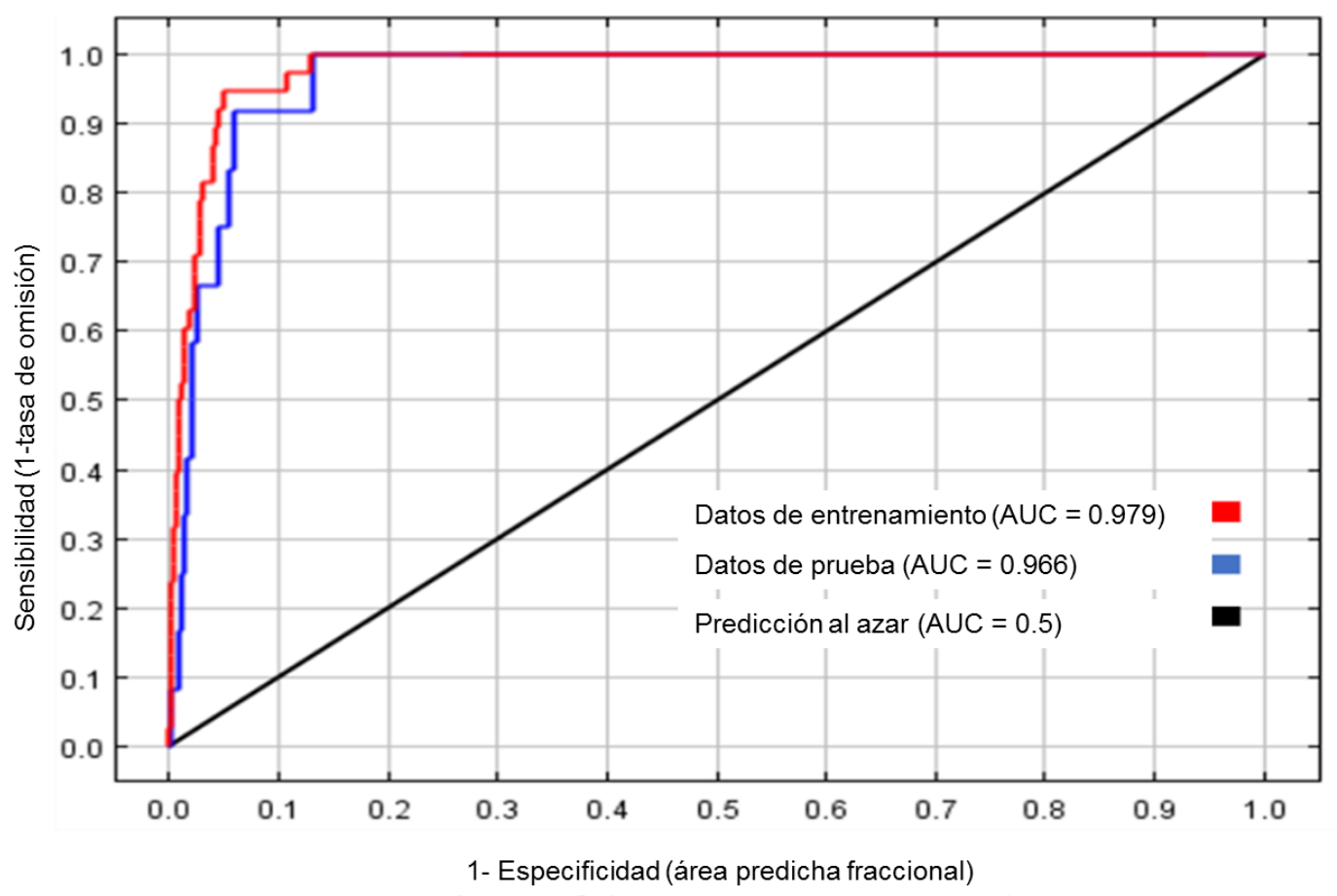

La Figura 2 muestra la distribución geográfica actual de T. diversifolia. Su presencia se encuentra principalmente en la región Centro-Sur, Golfo de México, las zonas costeras del Pacífico y la Península de Yucatán; es decir en la parte tropical del país ( $<23^{\circ} 26^{\prime} 14^{\prime \prime}$ Norte). La mayor parte de las poblaciones de $T$. diversifolia se concentran en la parte sureste de México, que está en correspondencia con la aseveración acerca de su origen geográfico

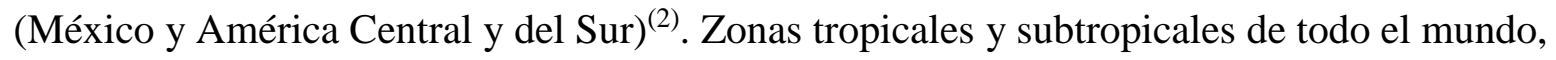
lo que le ha permitido, crecer bajo una amplia gama de condiciones ambientales $\mathrm{y}$, por tanto, desarrollar un amplio rango de adaptación ${ }^{(29)}$. 
Figura 2. Distribución actual, áreas aptas, no aptas, de retracción y expansión para $T$. diversifolia bajo cuatro escenarios de cambio climático en México
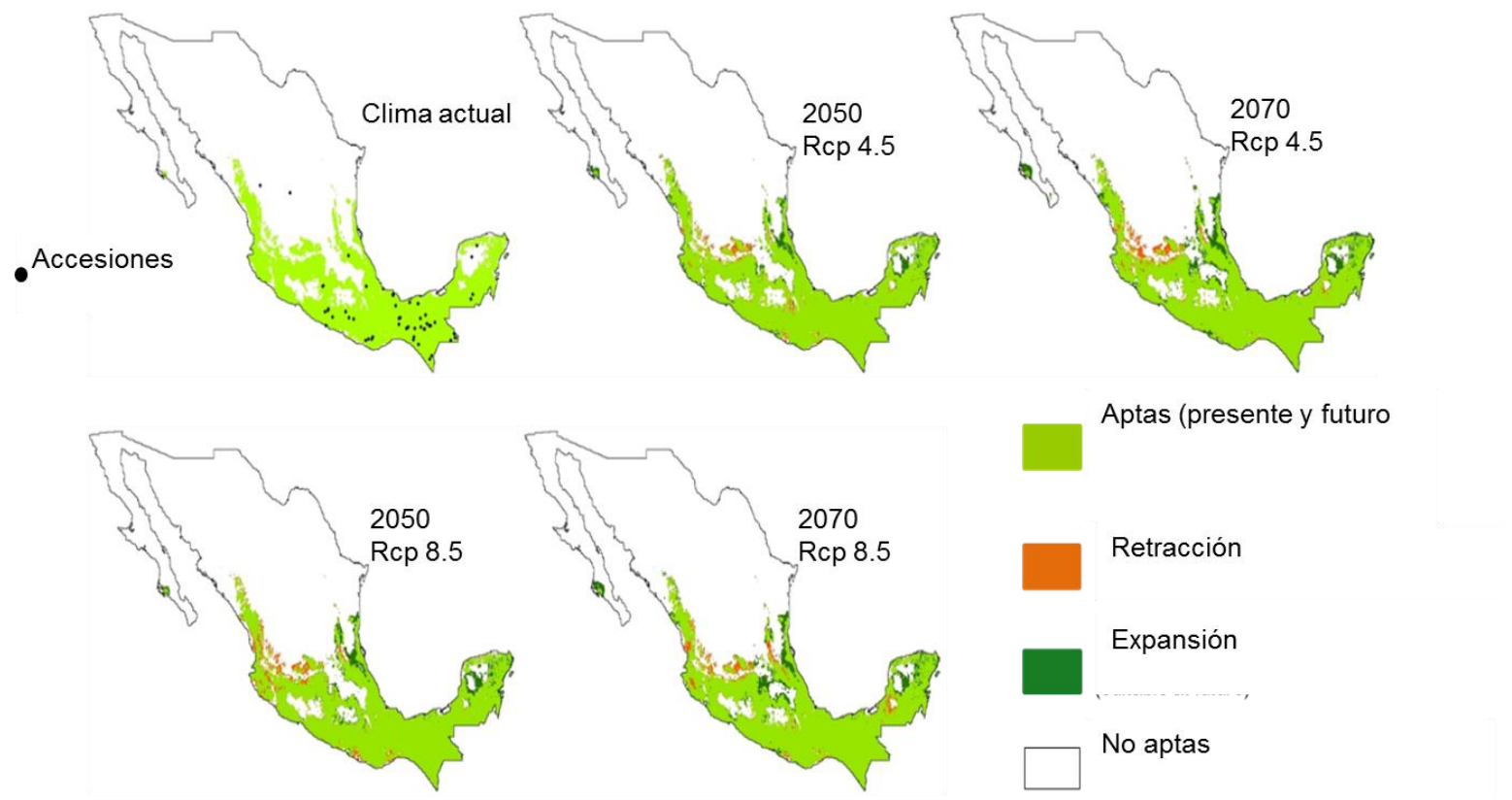

Los descriptores ecológicos de $T$. diversifolia y las variables ambientales que contribuyen a la construcción del modelo se pueden observar en el Cuadro 1. Según estos resultados, la distribución de $T$. diversifolia se determina principalmente por la variable pa5-10 (78.2 \%), esto es, la cantidad de precipitación durante el período mayo-octubre, la cual es un elemento clave para la presencia de esta especie, así como para otras especies, en donde la precipitación en pequeños pero frecuentes eventos durante el verano es clave para el buen desarrollo ${ }^{(30)}$. El descriptor de $T$. diversifolia para esta variable indica un rango de 297 a 3,404 mm (para la precipitación anual de 356 a 3,828 mm), lo que da una amplia variación de la precipitación espacial. Estos resultados son consistentes en el sentido que esta especie crece adecuadamente bajo una amplia gama de precipitación. Sin embargo, se han establecido límites de precipitación anual de 600 y $5,000 \mathrm{~mm}^{(30)}$. Por lo anterior, se puede concluir que T. diversifolia puede crecer incluso con menos precipitación estacional y anual de lo que indica la literatura actual.

El segundo descriptor ecológico más contribuyente fue txmc (temperatura máxima media del mes más cálido) y $T$. diversifolia también mostró tener un amplio rango térmico; 21.9 a 34.4 ${ }^{\circ} \mathrm{C}$. Teniendo en cuenta que $T$. diversifolia también tiene un amplio rango para el descriptor timf (temperatura media mínima del mes más frío, 1.8 a $19.8^{\circ} \mathrm{C}$, Cuadro 1) se puede concluir que esta especie es capaz de explorar y colonizar ambientes térmicamente extremos. Otros autores $^{(2)}$ habían indicado previamente que esta especie está adaptada a diferentes climas y altitudes. 
Cuadro 1. Descriptores ecológicos de las variables ambientales que condicionan en mayor medida la distribución geográfica de $T$. diversifolia

\begin{tabular}{lccc}
\hline Variable & Valor mínimo & Valor máximo & Contribución (\%) \\
\hline pa5-10, mm & 297 & 3404 & 78.2 \\
Txmc, ${ }^{\circ} \mathrm{C}$ & 21.9 & 34.4 & 5.9 \\
Pendiente, $\%$ & 0 & 14 & 3 \\
Tmmf, ${ }^{\circ} \mathrm{C}$ & 11 & 26.15 & 2.8 \\
Pmh, mm & 67 & 713 & 2.1 \\
\hline
\end{tabular}

Los resultados de los nichos potenciales para $T$. diversifolia bajo la climatología de referencia (1951-2000) así como las futuras climatologías, también se muestran en la Figura 2. Los mapas de esta figura muestran la presencia de áreas adecuadas, inadecuadas, de retracción y expansión para Tithonia diversifica en comparación con los escenarios de la climatología de referencia y las climatologías futuras. En todos los escenarios futuros aparecerán áreas de retracción, así como las áreas de expansión; mostrando la posible dinámica de las áreas con aptitud ambiental en función de los cambios climáticos futuros.

El umbral logístico de entrenamiento de presencia del percentil 10 estima un área con aptitud ambiental (para la climatología de referencia) que representa el $30.71 \%$ de la extensión territorial de México. Después de un balance entre las áreas de contracción y expansión de las climatologías de cambio climático, las áreas óptimas se incrementarán al $31.62 \%$, $31.83 \%, 32.45 \%$ y $32.45 \%$ del territorio mexicano, en los escenarios 2041-2060 Rcp8.5, 2041-2060 Rcp4.5 y 2061-2080 Rcp4.5, 2061-2080 Rcp8.5, respectivamente. Estos resultados muestran que los cambios climáticos futuros serán aparentemente benéficos para T. diversifolia. Al considerar que las variables más significativas en los modelos de los nichos de distribución son la precipitación acumulada de mayo a octubre y la temperatura máxima media del mes más cálido, se podría inferir que las combinaciones de estos parámetros bajo escenarios de cambio climático tendrán un efecto positivo en las áreas con aptitud ambiental para T. diversifolia.

En algunos estudios se ha señalado que el efecto del impacto del cambio climático sobre la distribución de las especies, pueden resultar en especies ganadoras y perdedoras ${ }^{(31)}$. El resultado final va a depender de su adaptación evolutiva. Por ejemplo, se han reportado expansiones de áreas adecuadas debido al cambio climático para otras especies como Leucospermum hypophyllocarpodendron subsp. hypophyllocarpodendron ${ }^{(11)}$.

La mayoría de los estudios sobre los efectos del cambio climático en la distribución de especies han reportado impactos negativos, siendo las áreas de retracción mayores que las áreas de expansión ${ }^{(32)}$. En el presente estudio se obtuvo lo contrario; es decir, áreas de expansión mayores que las áreas de retracción. Como se puede visualizar en la Figura 2, estos dos tipos de áreas tienen un patrón geográfico; las superficies de contracción se ubican 
principalmente en la región occidental del país y cerca de la costa del Pacífico, mientras que las de expansión se localizan principalmente en el lado oriental y cerca de la costa del Golfo de México. Este hecho apunta a dos regiones del país donde los futuros patrones climáticos difieren en las condiciones ambientales y, por lo tanto, difieren en el nivel de idoneidad para T. diversifolia. Esta situación en particular ya se ha manifestado, ya que la región correspondiente a las áreas de retracción ha sido reportada previamente con cambios en los patrones de cultivos como consecuencia de los cambios climáticos regionales ${ }^{(33,34)}$.

Aparentemente en los mapas que se ejemplifican en la Figura 2 ninguna población actual de T. diversifolia se ve afectada por la retracción de la aptitud ambiental. Sin embargo, en el caso de que algunas poblaciones de T. diversifolia se ubiquen en áreas de retracción en los próximos años, se debe considerar que estas poblaciones tendrán sólo dos opciones para sobrevivir: a) migrar a ambientes más favorables $o$; b) adaptarse a las nuevas condiciones climáticas ${ }^{(35)}$, que dependerá principalmente de la diversidad genética de las especies ${ }^{(36,37)}$. En el caso del presente estudio, se puede hipotetizar que la idoneidad medioambiental ganará un poco más de superficie debido al cambio climático, y esto podría aparentemente promover la futura dispersión de $T$. diversifolia a nuevas áreas. No obstante, es importante considerar las posibles capacidades de esta especie para competir contra otras del ecosistema o con especies invasoras que constituyen una amenaza para la estabilidad del ecosistema ${ }^{(38,39,40)}$. Además, es importante considerar que T. Diversifolia en el futuro también dependerá de su capacidad de adaptación al cambio climático ${ }^{(41)}$, lo que está en función de su capacidad para colonizar nuevas áreas o (cuando sea necesario) su capacidad de implementar modificaciones fisiológicas para adaptarse al nuevo entorno ${ }^{(42)}$. Al adaptarse al cambio climático y a los nuevos climas, la plasticidad ecológica de $T$. diversifolia ${ }^{(1,30)}$ constituye un aspecto clave $\mathrm{e}^{(43) \text {. }}$

De acuerdo con los resultados obtenidos, el cambio climático previsto para ambos períodos tendrá un efecto más positivo que negativo sobre la idoneidad ambiental de $T$. diversifolia (Figura 3), permitiendo así en el futuro su posible expansión territorial. Sin embargo, está claro que esta ventaja ambiental que será causada por el cambio climático futuro, resultará también favorable para otras especies tal vez en un contexto de competencia con $T$. diversifolia. Bajo este tipo de escenario, el amplio intervalo para la mayoría de los descriptores ecológicos de $T$. diversifolia (Cuadro 1) podría ser una característica ventajosa, aún más si se considera la diversidad genética de esta especie en otras partes del mundo, lo cual podría aumentar las capacidades de adaptación y colonización de T. diversifolia ${ }^{(36,44)}$. 
Figura 3. Dinámica del porcentaje de superficie con idoneidad ambiental para $T$. diversifolia en la climatología actual (presente) y cuatro climatologías futuras

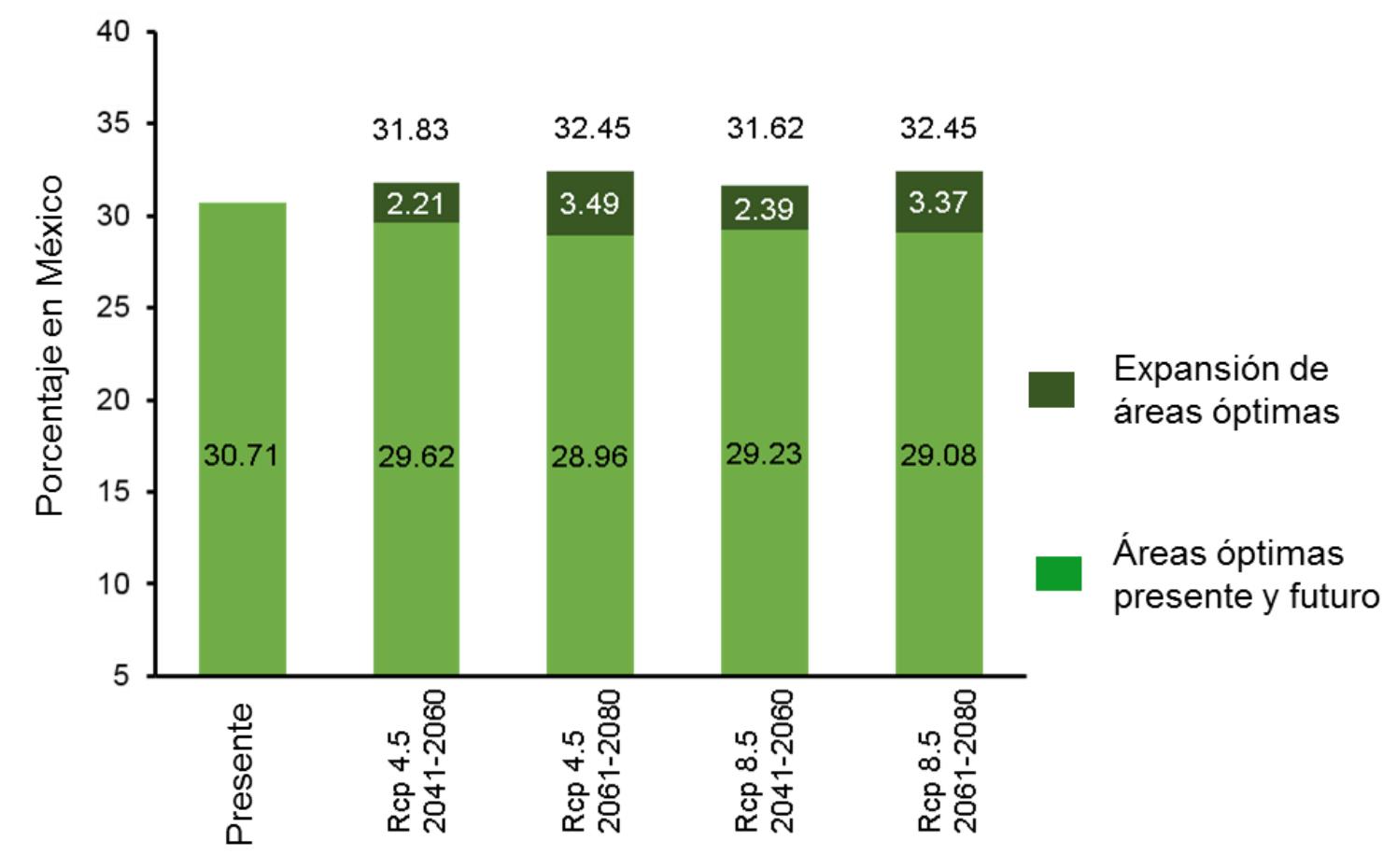

No obstante que los escenarios climáticos actual y futuros parecen ser favorables para $T$. diversifolia; deben adoptarse medidas para preservar las poblaciones actuales de $T$. diversifolia a fin de asegurar su presencia en su medio natural. Por otro lado, dado que se ha encontrado que $T$. diversifolia es una maleza alelopática con aleloquímicos solubles en agua en varias partes de la planta ${ }^{(17)}$, se debe prestar atención a supervisar su posible expansión territorial debido a posibles afectaciones a los cultivos asociados. También debe monitorearse la lucha contra la presencia de $T$. diversifolia como maleza en los cultivos, lo cual podría amenazar las poblaciones naturales de esta especie.

\section{Conclusiones e implicaciones}

Actualmente, las áreas con aptitud ambiental para $T$. diversifolia representan cerca de un tercio del territorio mexicano; adaptándose a una amplia gama de condiciones de temperatura y humedad. Su presencia y distribución espacial está determinada por la cantidad de precipitación acumulada durante el período mayo-octubre, con un rango de 297 a 3404 mm. Los cambios climáticos futuros provocarán tanto retracción como expansión de las áreas con aptitud ambiental en diferentes partes del país; haciendo un análisis entre estos dos efectos se concluye que en el presente siglo se incrementarán ligeramente estas áreas, entre 0.91 y $1.74 \%$ según los modelos utilizados. Por lo anterior, se puede considerar que esta especie puede ser una buena alternativa de producción de forraje para el futuro. Por otro lado, dado 
que se ha encontrado que $T$. diversifolia es una hierba alelopática, se debe poner atención en la supervisión de su posible expansión territorial debido a las posibles afectaciones a los cultivos asociados. La lucha contra la presencia de T. diversifolia en los campos de cultivo también debe supervisarse para que no constituya una amenaza para las poblaciones naturales de esta especie.

\section{Agradecimientos}

Al Inventario Nacional Forestal y de Suelos (INFYS) de la Comisión Nacional Forestal (CONAFOR), por brindar valiosa información sobre los sitios de presencia de T. diversifolia en México. También se agradece Instituto Nacional de Investigaciones Forestales Agrícolas y Pecuarias (INIFAP) por permitir el uso de las imágenes raster correspondientes a la altitud y pendiente del suelo del Sistema Nacional de Información Ambiental.

\section{Literatura citada:}

1. Pérez A, Montejo I, Iglesias JM, López O, Martín GJ, García DE, Milián I, Hernández A. Tithonia diversifolia (Hemsl.) A. Gray. Pastos y Forrajes 2009;32(1):1-15.

2. Loya OJL, Martínez GS, Prado ROF, Valdés GYS, Gómez, DAA, Escalera V F, Macedo BR, Durán PN. El Sistema Silvopastoril. Sistema Superior Editorial. Nayarit, México; 2014:102.

3. Intergovernmental Panel on Climate Change. Climate Change. The Physical Science Basis. Working group I. Contribution to the Fifth Assessment Report of the Intergovernmental Panel on Climate Change. Summary for Policymakers (Thomas FS, Dahe Q, Gian-Kasper P, Melinda MBT, Simon KA, Judith B, Alexander N, Yu X, Vincent B, Pauline MM. Cambridge University Press; 2013:29.

4. Milly PCD, Dunne KA, Vecchia AV. Global pattern of trends in stream low and water availability in a changing climate. Nature 2005;438(17):347-350.

5. William GJ. Causes of observed changes in extremes and projections of future changes. In: Thomas R. Karl, et al editors. Weather and climate extremes in changing climate regions of focus: North America, Hawaii, Caribbean and Pacific Islands, USA. A Report by the US Climate Change Science Program. Department of Commerce, NOAA's National Climatic Data Center, Washington, D.C., USA; 2008:81-116.

6. DeFries R, Rosenzweig C. Toward a whole-landscape approach for sustainable land use in the tropics. Proc Natl Acad Sci 2010;107:19627-19632. 
7. Gibbs HK, Ruesch AS, Achard F, Clayton MK, Holmgren P, Ramankuttly N, Foley JA. Tropical forests were the primary sources of new agricultural land in the 1980s and 1990s. Proc Natl Acad Sci 2010;107(38):16732-16737.

8. Foley JA, Asner GP, Heil C M, Coe MT, DeFries R, Gibbs HK, Howard EA, Olson S, Patz J, Ramankutty N, Snyder P. Amazonia revealed: forest degradation and loss of ecosystem goods and services in the Amazon Basin. Front Ecol Environ 2007;5(1):2532.

9. Schneider RR, Hamann A, Farr D, Wang, X, Boutin S. Potential effects of climate change on ecosystem distribution in Alberta. Can J For Res 2009;39:1001-1010.

10. Vale MM, Alves MAS, Lorini ML. Mudanças climáticas: desafios e oportunidades para a conservação da biodiversidade brasileira. Oecol Bras 2009;13:518-535.

11. Parmesan C. Ecological and evolutionary responses to recent climate change. Annu Rev Ecol Syst 2006;37:637-669.

12. Schneider RR, Hamann A, Farr D, Wang X, Boutin S. Potential effects of climate change on ecosystem distribution in Alberta. Can J For Res 2009;39:1001-1010.

13. Bellard C, Bertelsmeier C, Leadley P, Thuiller W, Courchamp F. Impacts of climate change on the future of biodiversity. Ecology Letters 2012;15:365-377.

14. Kirilenko AP, Belotelov NV, Bogatyrev BG. Global model of vegetation migration: incorporation of climatic variability. Ecological Modelling 2000;132:125-133.

15. Morueta HN, Fløjgaard C, Svenning JC. Climate change risks and conservation implications for a threatened small-range mammal species. PLoS ONE 2010;5(4):10360-101371.

16. Fasuyi AO, Dairo FAS, Ibitayo FJ. Ensiling wild sunflower (Tithonia diversifolia) leaves with sugar cane molasses. 2010. Livest Res Rural Develop 2015;42(22) from http://www.lrrd.org/lrrd22/3/fasu22042.htm.

17. Otusanya OO, Ilori OJ, Adelusi A.A. Allelopathic effects of Tithonia diversifolia (Hemsl) A. Gray on germination and growth of Amaranthus cruentus. Res J Environ Sci 2010;1(6):285-293.

18. Phillips SJ, Dudík M, Elith J, Graham CH, Lehmann A, Leathwick J, Ferrier S. Sample selection bias and presence-only distribution models: implications for background and pseudo-absence data. Ecological Applications 2009;19:181-197. 
19. Sobek S, Kluza S, Cuddington DAK, Lyons DB. Potential distribution of emerald ash borer: What can we learns from ecological niche models using Maxent and GARP? Forest Ecol Management 2012;281(1):23-31.

20. Moreno R, Zamora R, Molina JR, Vásquez A, Herrera M. Predictive modeling of microhabitats for endemic birds in South Chilean temperate forest using Maximum entropy (Maxent). Ecol Informat 2011;6:364-370.

21. Phillips SJ, Dudík M. Modeling of species distributions with Maxent: new extensions and a comprehensive evaluation. Ecography 2008;31:161-175.

22. Morales SN. Modelos de distribución de especies: Software Maxent y sus aplicaciones en conservación. Rev Conserv Ambi 2012;2(1):1-5.

23. Magaña RVO. Guía para generar y aplicar escenarios probabilísticos regionales de cambio climático en la toma de decisiones. Centro de Ciencias de la Atmósfera. Universidad Nacional Autónoma de México. México; 2010.

24. Díaz PG, Guajardo PRA, Medina GG, Sánchez CI, Soria RJ, Vázquez AMP, et al. Potencial productivo de especies agrícolas de importancia socioeconómica en México. 1a. ed. INIFAP. Xalapa, Ver., México; 2012.

25. Eastman JR. Idrisi Selva Manual, Version 17. Clark Labs, Clark University. Worcester, Mass, USA; 2012.

26. Escalante T, Rodríguez TG, Linaje M, Illoldi RP, González LR. Identification of areas of endemism from species distribution models: threshold selection and nearctic mammals. Rev Especializada Cienc Químico-Biol 2013;16(1):5-17.

27. Instituto Nacional de Estadística Geografía e Informática (INEGI). Guía para interpretación cartográfica: Uso de suelo-vegetación Serie III. DF., México. 2009.

28. Parolo G, Rossi G, Ferrarini A. Toward improved species niche modelling: Arnica montana in the Alps as a case study. J Appl Ecol 2008;45(5):1410-1418.

29. Chapin III FS, Bloom AJ, Field CB, Waring RH. Plant responses to multiple environmental factors. BioScience 1987;37(1):49-57.

30. Murgueitio E, Rosales M, Gómez ME. Agroforestería para la producción animal sostenible. Centro para la Investigación en Sistemas Sostenibles de Producción Agropecuaria. Cali, Colombia; 2001.

31. Hoffman AA, Sgró CM. Climate change and evolutionary adaptation. Nature 2011;470:479-485. 
32. Sork V, Davis F, Westfall R, Flints A, Ikegami M, Wang H, Grivet DD. Gene movements and genetic association with regional gradients in California valley oak (Quercus lobata Née) in the face of climate change. Mol Ecol 2010;19:3806-3823.

33. Ramírez LMR, Ruiz CJA, Medina GG, Jacobo CJL, Parra QRA, Ávila MMR, Pilar AJ. Perspectivas del sistema de producción de manzano en Chihuahua, ante el cambio climático. Rev Mex Cienc Agr 2011;(Pub Esp 2):265-279.

34. Santillán ELE, Blanco MF, Magallanes QR, García HJL, Cerano PJ, Delgadillo RO, Valdez CRD. Tendencias de temperatura extremas en Zacatecas, México. Rev Mex Cienc Agr 2011;( Pub Esp 2):207-219.

35. Jump AS, Peñuelas J. Running to stand still: Adaptation and the response of plants to rapid climate change. Ecology Letters 2005;8:1010-1020.

36. Burke MB, Lobell DB, Guarino L. Shifts in African crop climates by 2050, and the implications for crop improvement and genetic resources conservation. Global Environmental Change 2009;19(3):317-325.

37. Mercer KL, Perales HR. Evolutionary response of landraces to climate change in centers of crop diversity. Evol Appl 2010;3(5-6):480-493.

38. Hellmann JJ, Byers JE, Bierwagen BG, Dukes JS. Five potential consequences of climate change for invasive species. Conservation Biol 2008;22(3):534-543.

39. Howard G, Ziller SR. Alien alert: Plants for biofuel may be invasive. Bioenergy Business 2008:14-16.

40. Mainka SA, Howard GW. Climate change and invasive species: Double jeopardy. Integrative Zoology 2010;5:102-111.

41. Alsos IG, Alm T, Normand S, Brochmann C. Past and future range shifts and loss of diversity in dwarf willow (Salix herbacea L.) inferred from genetics, fossils and modelling. Global Ecol Biogeogr 2009;18:223-239.

42. Chown SL, Hoffman AA, Kristensen TN, Angilletta Jr, Stenseth MJ, Pertoldi NCh, C. Adapting to climate change: a perspective from evolutionary physiology. Climate Res 2010;43:3-15.

43. Peters K, Breitsameter L, Gerowitt B. Impact of climate change on weeds in agriculture: a review Agron Sustain Dev 2014;34:707-721.

44. Ruiz CJA, Hernández CJM, Sánchez GJJ, Ortega CA, Ramírez OG, Guerreo HMJ, et al. Ecología, adaptación y distribución actual y potencial de las razas mexicanas de maíz. Libro Técnico Núm. 5. INIFAP-CIRPAC-Campo Exp Centro Altos de Jalisco. 2013. 